\title{
LA CANASTA DE CONSUMO DE LOS HOGARES DOMINICANOS, SU EVOLUCIÓN HISTÓRICA Y LOS POSIBLES CAMBIOS DERIVADOS DE LA PANDEMIA DEL COVID-19
}

\author{
IsIDORO SANTANA $^{1}$
}

Recibido: 10/04/2021 - Aceptado: 01/09/2021

Cómo citar: Santana, I. (2021). La canasta de consumo de los hogares dominicanos, su evolución histórica y los posibles cambios derivados de la pandemia del COVID-19. Ciencia, Economía y Negocios, 5(2), 129-149. Doi: https://doi.org/10.22206/ceyn.2021.v5i2.pp129-149

\section{Resumen}

Este artículo muestra la estructura de consumo de la población dominicana antes de la pandemia, a partir de datos de la ENGIH-2018, los cambios de la canasta desde la primera ENGIH en 1976-1977 y, finalmente, se emite una idea de los posibles efectos de la pandemia sobre esta. En la estructura de consumo familiar influyen factores como nivel de ingresos, urbanización, tamaño de los hogares, estratificación social, educación, etc. A su vez, en una economia bastante cerrada, la estructura de consumo es determinante de las estructuras de producción, empleo, comercio, ingresos fiscales, comercio exterior y condiciones sociales. Los hábitos de gastos cambian con el tiempo y las circunstancias. En medio siglo, la economía dominicana ha crecido, han surgido nuevos sectores y la población se ha urbanizado. Con esto, ha cambiado la canasta hacia una menor ponderación del componente alimentación y de prendas de vestir, y el consiguiente incremento del consumo de servicios, incluyendo transporte, educación, salud y comunicaciones. Dentro de la alimentación, han ganado terreno los alimentos servidos fuera del hogar. La pandemia del COVID-19 impactó de manera aguda los modos de vida y es previsible que los hábitos de consumo de la gente, impactando a su vez la estructura productiva y de empleo.

Palabras clave: canasta de alimentos, consumo, COVID-19, ENGIH.

Código de JEL: D12, D15, D60.

\footnotetext{
${ }^{1}$ Escuela de Economía \& Negocios, Instituto Tecnológico de Santo Domingo (INTEC), Santo Domingo, República Dominicana. Correo-e: isidoro.santana@gmail.com
} 


\title{
CONSUMPTION BASKET OF DOMINICAN HOUSEHOLDS: HISTORICAL EVOLUTION AND POSSIBLE CHANGES DERIVED FROM THE COVID-19 PANDEMIC
}

\author{
IsIDORo SANTANA
}

Received: 04/10/2021 Approved: 09/01/2021

\begin{abstract}
This article shows the household consumption structure of the Dominican Republic before the pandemic, based on data from the ENGIH-2018; the changes of the basket since the first ENGIH in 1976-1977 and, finally, issues an idea of the possible effects of the pandemic on it. The structure of family consumption is influenced by factors such as income level, urbanization, household size, social stratification, education, etc. In turn, in a fairly closed economy, the structure of consumption is determinant of the structures of production, employment, trade, tax revenues, foreign trade and social conditions. Spending habits change with time and circumstances. In half a century, the Dominican economy has grown, new sectors have emerged, and the population has urbanized. With this, the consumption basket has changed towards a lower weighting of the food and clothing component, and consequent increase in the consumption of services, including transportation, education, health, and communications. Within the food, food served outside the home has gained ground. Since 2020, many things have changed, as the COVID-19 pandemic has acutely impacted lifestyles, and it is foreseeable that people's consumption habits will be affected and, with them, the productive and employment structure will be impacted.
\end{abstract}

Keywords: Consumption basket, COVID-19, goods and services, ENGIH.

JEL Codes: D12, D15, D60. 


\section{Introducción}

A partir del año 2020 muchas cosas han cambiado en el mundo. Además de los sistemas de salud, elemento primigenio, y de la macroeconomía, primera derivación, la pandemia del COVID-19 impactó de manera aguda los sistemas educativos, las estructuras sociales, los modos de vida, las relaciones humanas (incluso familiares), la política y la psicología colectiva. Es muy previsible que los hábitos de consumo de la gente también hayan cambiado.

El presente artículo se propone mostrar, en primer lugar, los niveles de ingreso y la estructura de consumo de la población dominicana antes de la pandemia, a partir de la Encuesta Nacional de Gastos e Ingresos de los Hogares del 2018 (ENGIH-2018), ejecutada por el Banco Central (Banco Central de la República Dominicana, 2018); en segundo lugar, los cambios que han venido sucediendo a lo largo del tiempo desde la primera ENGIH en 1976-1977 y, finalmente, emitir una idea de los posibles efectos de la pandemia sobre la estructura de la canasta de consumo.

La estructura de consumo es determinante de las estructuras de producción, de comercio, de ingresos fiscales, de importaciones e inversiones. Consiguientemente, del empleo y de las condiciones sociales. Dado que la República Dominicana tiene un coeficiente de exportaciones de bienes muy bajo, un 12.6\% del PIB en 2019 (antes del COVID-19), y sigue bajo, aun incorporando las exportaciones de servicios (23\%, frente a un promedio mundial de $31 \%$ ); es razonable entender que la estructura de producción fue construida básicamente para responder a la demanda interna, en lo fundamental a la estructura de consumo.

Por tanto, los cambios en los hábitos de consumo impactan la producción y el empleo, pues todo bien o servicio dejado de consumir, como puede haber ocurrido en 2020, significa negocios cerrados, empleos e ingresos perdidos, con repercusiones para los demás negocios. El mismo razonamiento aplica al revés, para los nuevos bienes y servicios incorporados a la canasta familiar.

\section{Estructura del consumo familiar en el año 2018}

En la República Dominicana, el gran precursor y artífice de las estadísticas socioeconómicas y de las encuestas sobre presupuestos familiares 
fue Gumersindo del Rosario. Bajo su dirección, el Banco Central realizó dos encuestas nacionales de este tipo: la primera hace más de cuatro décadas, entre mayo de 1976 y abril de 1977 y la segunda en noviembre de 1984. Anterior a esta hubo otra, en 1969, pero limitada a los hogares residentes en la ciudad de Santo Domingo, de modo que sus datos no son extrapolables al resto del país, y más que en ese tiempo todavía esa ciudad era poco representativa de la población dominicana. Después se hizo una tercera ENGIH entre octubre de 1997 y septiembre de 1998, una cuarta en 2007 (dirigida por la Oficina Nacional de Estadística) y, finalmente, la quinta en el ańo 2018. De los resultados de las primeras encuestas, Del Rosario sustentó su estudio Estructura de Consumo, Nutrición y Pobreza en la República Dominicana, 1984 (Del Rosario, 1984).

La Figura 1 muestra la estructura de consumo de los hogares dominicanos conforme a la última medición realizada, es decir, correspondiente al año 2018, cuando todavía no había pandemia. En la estructura de consumo influyen múltiples factores, incluyendo el nivel de ingresos, la urbanización, el tamaño de los hogares, la estructura social, el nivel educativo, la cultura, el efecto demostración transmitido por los medios de comunicación o las interacciones con otras sociedades por vías de turismo, migración, etc., y todos esos elementos cambian con el tiempo y las circunstancias.

Por tanto, es lógico suponer que cada hogar tiene su propia canasta y lo que se extrae de las encuestas de hogares no es más que promedios nacionales o, a lo sumo, promedios por grupos amplios, como zonas o regiones geográficas, quintiles o deciles de ingresos, niveles educativos, etc. 


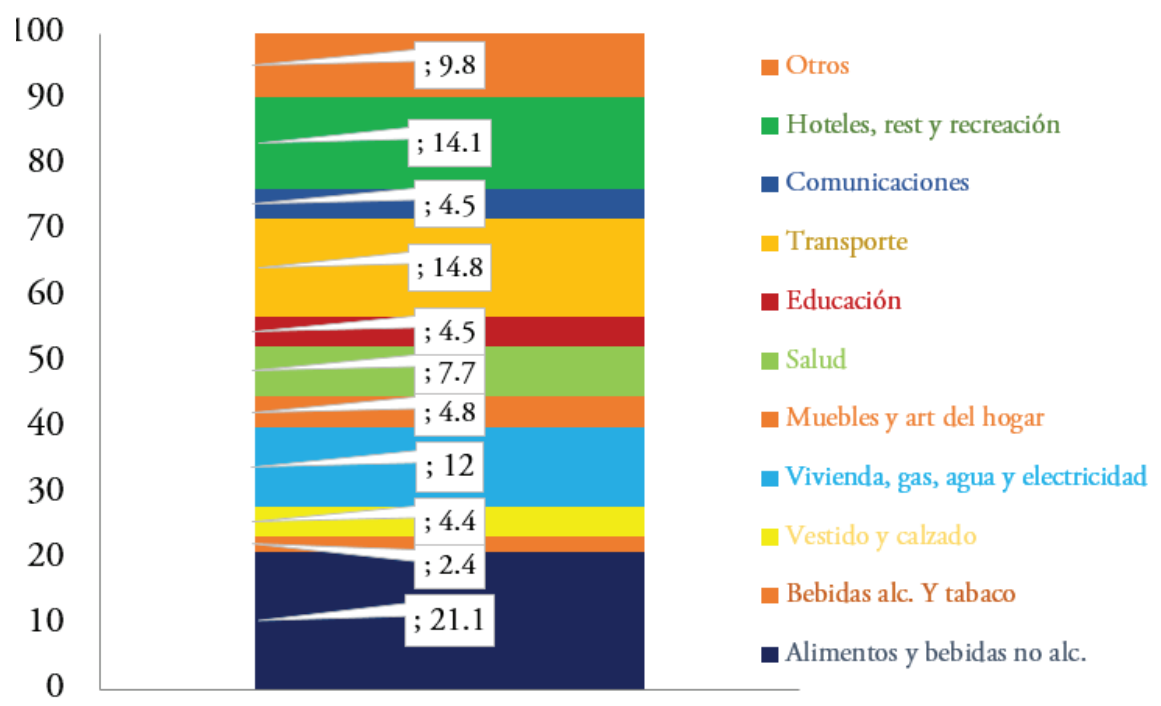

Figura 1. Estructura de la canasta promedio de consumo de los hogares dominicanos, año 2018

Notas. Cifras en porcentaje del monto total, el cual está en base a DOP 33,844 mensuales. Los cálculos incluyen el autoconsumo, excepto en el concepto de vivienda (alquiler imputado). Gráfico elaborado por el autor en base a la ENGHI-2018 del BCRD.

Como se aprecia, el monto promedio ascendió a RD $\$ 33,844$ mensuales en el año 2018. Ahora bien, es importante anotar que ese monto, en que el peso fundamental se explica por el valor gastado en los componentes alimentos y bebidas, servicios de transporte, hoteles y restaurantes, así como vivienda y los principales servicios del hogar, se calculó incluyendo el autoconsumo, excepto el de la vivienda.

Si se incluyera en la canasta el servicio del alquiler imputado, entonces el valor total de esta ascendería a $\mathrm{RD} \$ 38,119$ mensuales por hogar, el peso del componente vivienda sería mucho más elevado y, consecuentemente, bajaría el peso de los demás. Más adelante trataremos de exponer las implicaciones de esto.

Probablemente el principal determinante de la canasta es el nivel de ingreso de los hogares, el cual se muestra a continuación por grandes regiones geográficas (Figura 2). En la figura pueden apreciarse las notorias diferencias en función del lugar en que reside el hogar y cómo la 
media de ingresos en todas las regiones fuera de la capital está por debajo del promedio nacional (44,748 pesos mensuales), lo que indica que el nivel de ingresos del Gran Santo Domingo es el que empuja hacia arriba la media.

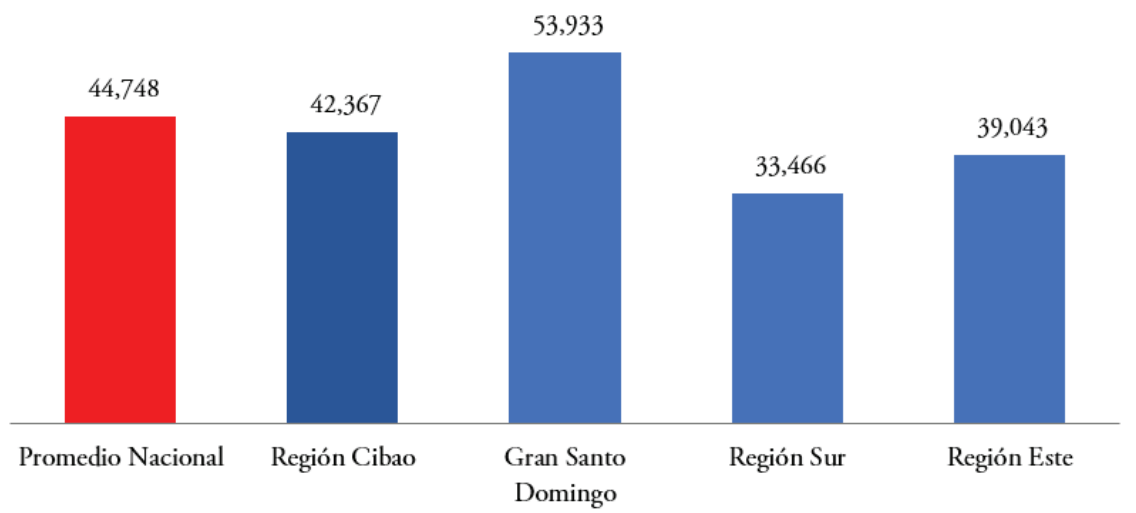

Figura 2. Ingreso promedio mensual de los hogares dominicanos por macroregiones geográficas, 2018

Notas. Cifras en pesos dominicanos (DOP). Cálculos incluyen alquiler imputado. La figura fue elaborada por el autor en base a la ENGHI-2018 del BCRD.

Esto también está vinculado a la urbanización, pues los ingresos medios son más elevados en la zona urbana que en las rurales (en promedio, los superan en 50\%) y, obviamente, Santo Domingo es la más urbanizada de las macrorregiones. De hecho, eso mismo explica que en la Región Norte o Cibao los ingresos familiares sean algo mayores que en el Este y el Sur.

Si bien la canasta media está diferenciada por zonas o regiones de residencia, el factor diferenciador principal es el nivel de ingreso de los hogares, el cual es mostrado en el gráfico siguiente por grupos quintílicos. El ingreso del $20 \%$ más alto supera, en promedio, en 6.6 veces el ingreso correspondiente al quintil más bajo.

Observar que el quintil llamado medio alto de ninguna manera puede asimilarse a lo que suele denominarse clase media alta, pues el ingreso medio de este grupo apenas sumaba $\mathrm{RD} \$ 43,848$ mensuales por hogar en el año 2018. La indicada clase media alta está incluida en el quintil supe- 
rior, que incluye alrededor de 643 mil familias; en sociedades como la dominicana, la denominación de clase alta está reservada a algunos miles de familias, por lo que el quintil superior representa mucho más que ese limitado grupo.

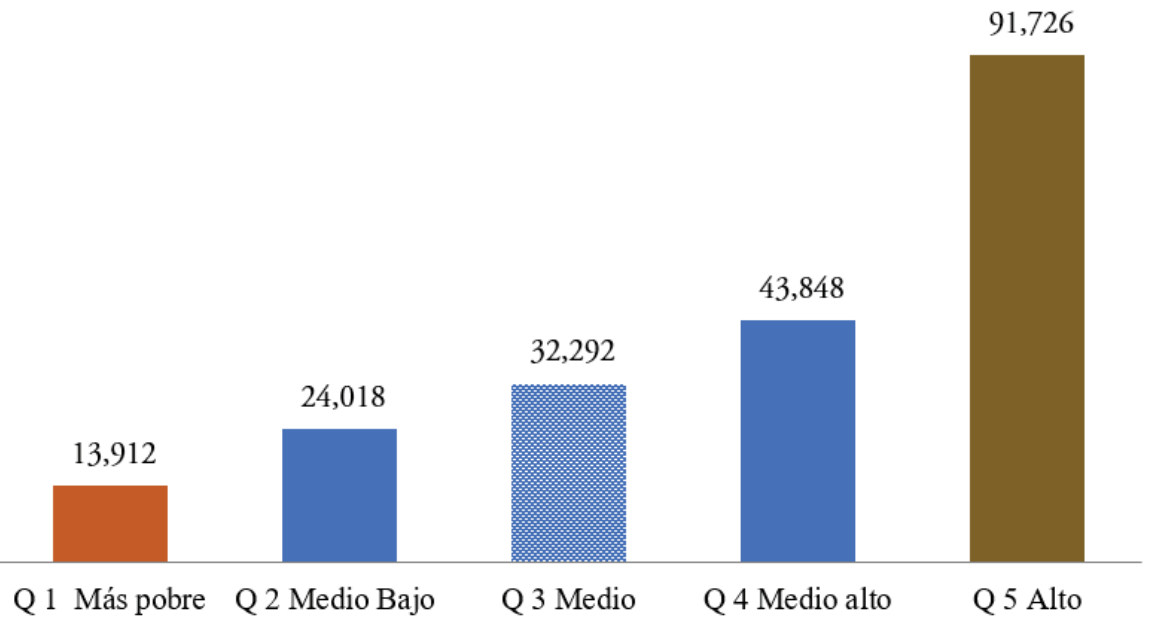

Figura 3. Ingreso promedio mensual de los hogares dominicanos por quintiles de ingreso mensual, 2018

Notas. Cifras en pesos dominicanos (DOP). Cálculos incluyen alquiler imputado. La figura fue elaborada por el autor en base a la ENGHI-2018 del BCRD.

\section{Cambios en el tiempo de la estructura de consumo familiar}

De los datos resultantes de las cinco ENGIH se pueden inferir transformaciones importantes en la estructura de consumo de las familias, en algunos casos congruentes con una mayor modernización y progreso, y en otros casos para señalarnos fallos de política que por tiempo han condicionado los costos de vida para los ciudadanos.

Como advertíamos en principio, la canasta de consumo de las familias depende no solo de la economía, sino también de la estructura social, la cultura, de influencias externas e incluso de factores como el confinamiento. Pero es evidente que a largo plazo la economía es el determinante 
fundamental, por lo que las variaciones en el nivel de ingreso influyen sobremanera.

La primera encuesta nacional de gastos e ingresos de las familias se realizó hace más de cuatro décadas, entre 1976 y 1977. En aquel tiempo, como corresponde a una economía de bajos ingresos, la mayor parte del presupuesto de las familias dominicanas se destinaba a la alimentación, preponderante en el concepto "alimentos, bebidas y tabaco".

La alimentación es, con mucho, la más primaria de las necesidades humanas, por lo que a bajos niveles de ingreso, esta absorbe una gran proporción del ingreso, la que va bajando conforme se incrementa el ingreso. Esto había sido documentado estadísticamente desde 1857 por Ernst Engel mediante un estudio referido a Bélgica (Engel, 1895), de donde derivó la Ley de Engel, según la cual el gasto en alimentos es una función creciente del ingreso y del tamaño del hogar, pero el porcentaje de gasto en alimentos disminuye con el ingreso. De ahí que en las sociedades de bajos niveles de ingreso una parte muy grande de los gastos familiares sea absorbida por la alimentación.

Así que en ese tiempo resultó que los hogares gastaban en el renglón alimenticio el $50.8 \%$ de sus presupuestos. Para 1984 ese porcentaje se había reducido al 42.8\%, y así sucesivamente, hasta que ya en la más reciente encuesta, que tuvo lugar en 2018, había bajado a 32.2\%. Una parte de las diferencias observadas en la estructura de gastos se vinculan con algunos cambios metodológicos realizados en el tiempo principalmente en la definición de los conceptos y en el procesamiento y la difusión de las encuestas. Los más importantes, que tendremos que explicar, son los relativos al gasto en alimentación y al componente vivienda (Figura 4). 


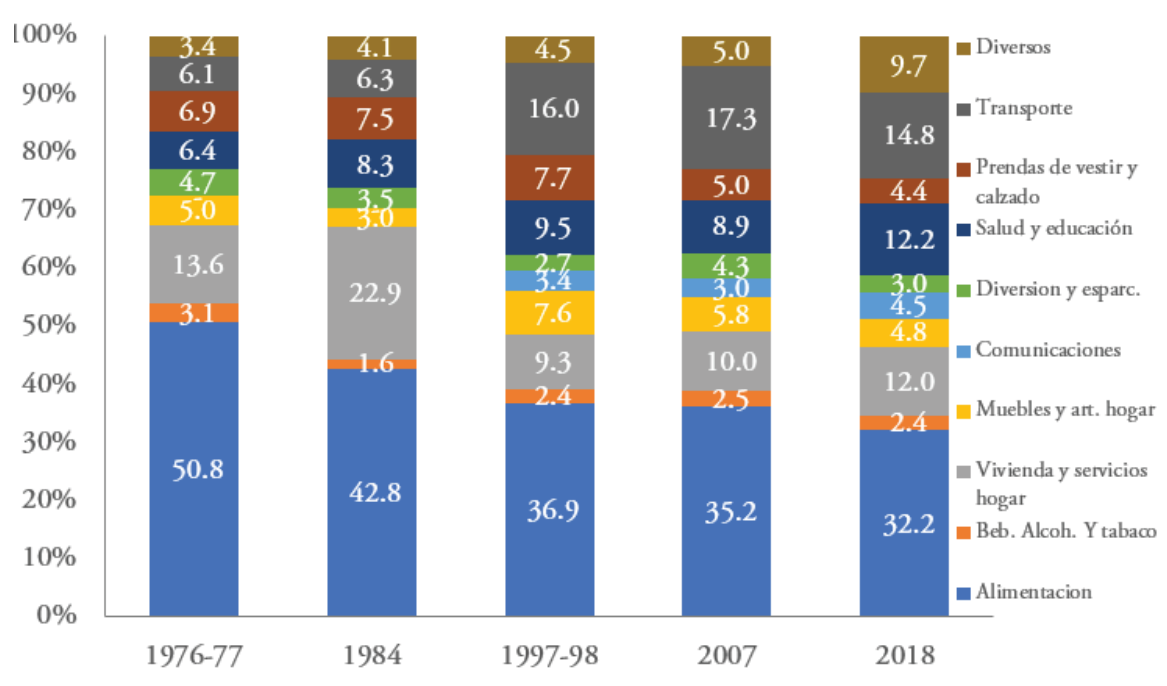

Figura 4. Evolución de la estructura de los presupuestos familiares, $1976-77$ a 2018

Notas. (1) El concepto de vivienda, a partir de 1997-98, no incluye el autoconsumo (alquiler imputado). (2) En el concepto alimentación se incluyen los alimentos preparados fuera de la casa (llamado a partir de la ENGIH 1997-98, "hoteles y restaurantes"). La figura fue elaborada por el autor en base a la ENGHI-2018 del BCRD.

\section{El caso del consumo en alimentos}

En las cifras publicadas por el Banco Central, la reducción del componente alimentos aparece mucho más radical, pues en 2018 solo representa un $21.1 \%$ del consumo total, en vez del $32.2 \%$ que se muestra aquí (Figura 5).

La diferencia obedece, fundamentalmente, al siguiente fenómeno social. Al hacerse la primera encuesta (1976-77), aparecía una pequeña porción denominada alimentos preparados fuera del hogar, pero aparentemente el porcentaje resultante era tan pequeño que ni siquiera se incluyó explícitamente en la publicación. En la segunda encuesta (1984) se volvió a medir, resultando que un $2.2 \%$ de los gastos familiares se hacían en alimentos servidos fuera de la casa, pero incluidos en el componente alimenticio (42.8\%). 
En la medida que la sociedad fue cambiando, por múltiples razones este porcentaje de alimentos servidos fuera de la casa se hizo más grande, y ya para la tercera encuesta dejó de incluirse en el componente alimentación y se pasó a publicar un nuevo concepto, denominado hoteles y restaurantes, con un 7.2\% (Banco Central de la República Dominicana, 1999).

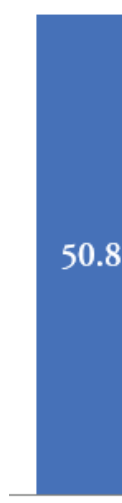

1976
— Hoteles y restaurantes

Alimentos y bebidas en el hogar

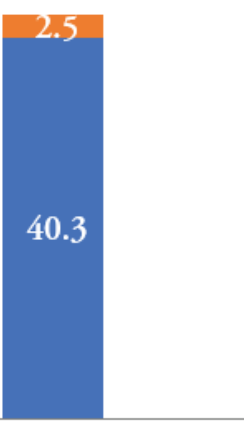

1984

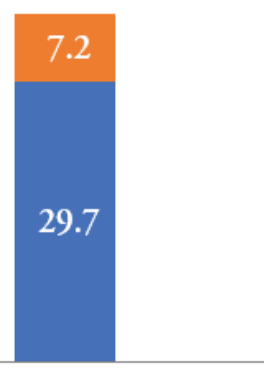

1998

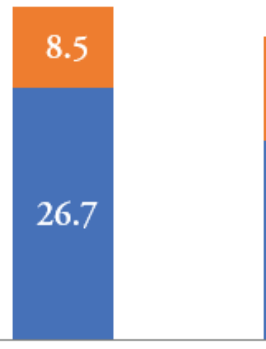

2007
11.1

21.1

2018

Figura 5. Porcentaje de presupuesto familiar destinado a alimentación, incluidos los alimentos fuera del hogar

Notas. (1) Para mantener uniforme la secuencia con publicaciones anteriores, hemos excluido las bebidas alcohólicas y el tabaco en cada caso. (2) Al cambiarse la denominación de alimentos "consumidos fuera del hogar", a partir del 1997-1998, por "hoteles y restaurantes", es posible que incluya una pequeña fracción no alimenticia. La figura fue elaborada por el autor en base a la ENGHI-2007 y encuestas del BCRD.

La denominación no hace honor al contenido, pues la expresión hoteles y restaurantes remite a la idea de un consumo suntuario, propio de la clase media y alta, pero en realidad lo que es propiamente hoteles es una ínfima parte, y más del $90 \%$ corresponde a la comida que muchos obreros y empleados consumen en establecimientos que ni siquiera llegan a la categoría de restaurantes, sino que son fondas, comedores, lugares de expendio, incluso ambulantes, o sencillamente comida adquirida o suministrada en los propios centros de trabajo. 
No por casualidad, contrario a lo que se esperaría, los datos muestran que los pobres gastan una porción mayor de sus presupuestos que los ricos en el concepto de hoteles y restaurantes, $(12.1 \%$ el quintil más bajo frente a $9.3 \%$ el quintil superior), como se ilustra en el siguiente gráfico, con la composición de la canasta correspondiente a los dos extremos de la estratificación social (quintiles I y V). Para evitar confusión en las comparaciones en el tiempo, para este artículo hemos optado por sumarlo nuevamente en el componente alimenticio.

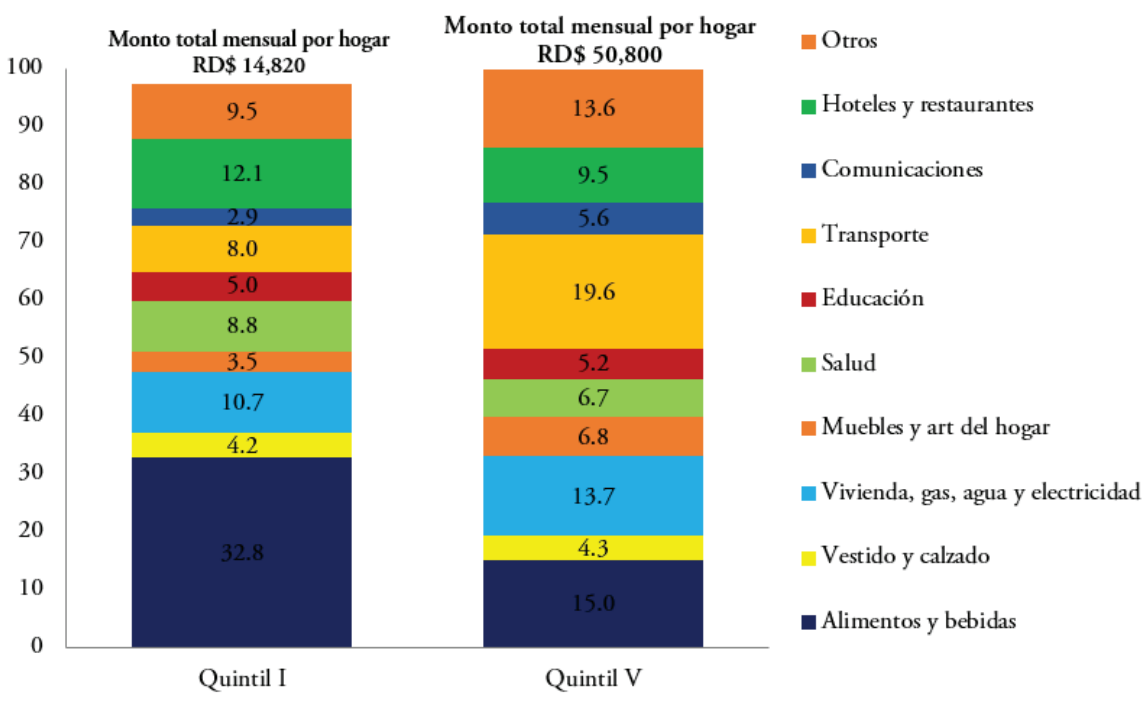

Figura 6. Estructura de la canasta de gastos de los hogares dominicanos en Quintiles I y V, 2018

Notas. En porcentaje del monto total. Figura elaborada por el autor en base a la ENGHI-2018 y encuestas del BCRD.

Con los datos del año 2018, el componente llamado hoteles y restaurantes representa cerca de la tercera parte del presupuesto de alimentación, debido fundamentalmente a que, por el crecimiento de las ciudades, el congestionamiento del tránsito y el encarecimiento del transporte, se dificulta moverse a comer a la casa, a lo que contribuyen factores como la abundancia de negocios de comida rápida o el establecimiento de comedores en centros de trabajo, la integración de la mujer al mercado laboral, 
que reduce las posibilidades de dedicarse a preparar las comidas en la casa, entre otros.

Recientemente, en el año 2021, fue publicado por el Instituto Dominicano de Investigaciones Agroforestales (IDIAF), un estudio del investigador Pedro Juan del Rosario, denominado El Consumo de Alimentos en la República Dominicana, en el cual hace un minucioso análisis de los cambios que han venido teniendo lugar en los hábitos de consumo alimenticio de la población y los factores que influyen en esto. Explica una tendencia de largo plazo a aumentar el consumo de alimentos de origen animal sobre los de origen vegetal.

Comparando las últimas dos encuestas de presupuestos familiares, encuentra "un incremento de la participación de a) leche, quesos y huevos, b) legumbres-hortalizas, c) frutas, d) carnes y pescado y, e) café y cacao. En cambio, hay una reducción relativa en los grupos: a) pan y cereales, b) aceites y grasas, c) azúcares y mermelada, d) gaseosas y jugos envasados y, e) preparados alimenticios, condimentos y aderezos procesados" (Del Rosario, P. J., 2021).

\section{El gasto en viviendas}

Otra diferencia metodológica importante se observa en la vivienda. Anteriormente, en las primeras encuestas, algunos elementos de la estructura de consumo se consideraban dentro del renglón vivienda. Tal es el caso de la partida "mobiliario y equipo doméstico", posteriormente separado y denominado "muebles y artículos del hogar". Sin embargo, para los fines de este artículo, la disposición de los datos nos permitió extraerla de las primeras encuestas y uniformar la serie. Diferente es el caso de los servicios de comunicaciones (el teléfono era un servicio domiciliario), que se mantiene como "vivienda" en las primeras dos encuestas y después fue separado como un renglón aparte.

Ahora bien, la diferencia más importante proviene del tratamiento del autoconsumo, es decir, el alquiler imputado. A diferencia de la alimentación, en el presupuesto promedio de las familias el componente de vivienda, incluyendo servicios del hogar como agua, electricidad y gas, tendía a ganar ponderación con el progreso económico. En 1976-77 los hogares gastaban el $13.6 \%$ de sus presupuestos en este concepto y en 1984 el 22.9\%. A partir de 1998 se dejó de incluir el alquiler imputado cuando 
la vivienda es habitada por su propietario o en calidad de cedida o prestada. De este modo, volvió a bajar el gasto en vivienda, denominado recientemente alojamiento, agua, electricidad, gas y otros combustibles, el que representó en 2018 un 12\% del presupuesto familiar.

Aparentemente la motivación principal de no incluir el alquiler imputado por el servicio de viviendas es que el Banco Central, ejecutor de la Encuesta Nacional de Gastos e Ingresos de los Hogares, utiliza estos datos para el cálculo posterior del Índice de Precios al Consumidor (IPC) y de la tasa de inflación; metodológicamente resulta difícil incorporar en el cálculo de la inflación un elemento importante cuyas variaciones es imposible medir objetivamente, a diferencia de aspectos como una libra o unidad de algún alimento o servicio.

Si hacemos un ejercicio en el cual se incluye el alquiler imputado en el presupuesto del hogar, entonces el monto de la canasta media se eleva a $\mathrm{RD} \$ 38,119$ pesos mensuales (en 2018), el componente vivienda sube al $20 \%$ y todos los demás bajan su participación en el total, como se aprecia a continuación.

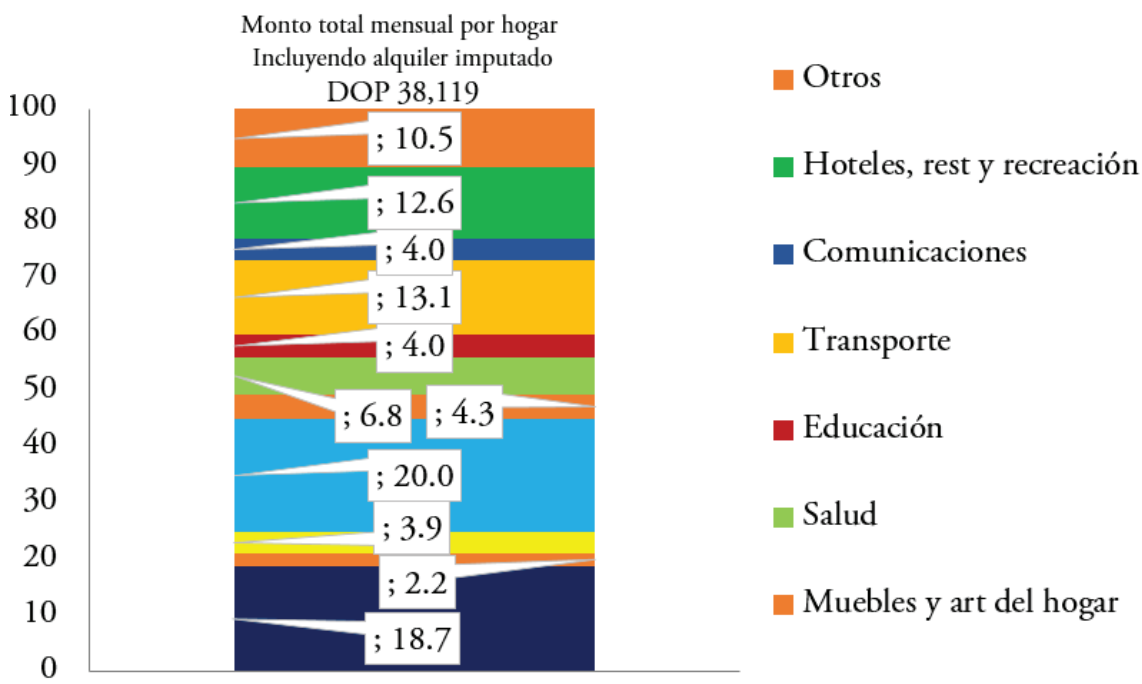

Figura 7. Estructura de la canasta promedio de gastos de los hogares dominicanos, 2018

Notas. En porcentaje del monto total. La figura fue elaborada por el autor en base a la ENGHI-2018. 
Ciertamente, la decisión de no incluir el alquiler imputado por vivir en casa propia o usada gratuitamente puede ser positivo para medir la inflación, pero puede afectar mucho el análisis cuando se trata de medir gastos o ingresos familiares. Curiosamente, en el análisis del Banco Central el alquiler imputado sí es incluido en la medición del ingreso familiar, pero no en la canasta de consumo. Macroeconómicamente, esto puede ser fuente de incompatibilidades para la generación de las cuentas nacionales. Para que tengamos una idea de su trascendencia, en la medición de los ingresos el alquiler imputado representa el 8.2\% del total nacional; y esto es fácil de explicar, pues solo el 35.7 de los hogares vive en viviendas alquiladas, de modo que habría que imputarle el ingreso y el gasto por alquiler al $64.3 \%$ restante.

Un ejemplo sencillo de los problemas que esto acarrea: si dos hogares reciben igual ingreso y ejecutan igual monto de gasto monetario, parecería que tienen igual nivel de vida; pero si uno de los dos vive en casa propia mientras el otro paga alquiler, no hay dudas de que el primero tiene un consumo más elevado que el segundo, lo que se oculta al excluir el alquiler imputado.

Otra deformación puede resultar del ejemplo siguiente: dos hogares perciben sus ingresos por sumas similares y ambos viven en casas propias; por tanto, ninguno paga alquiler y sus ingresos y gastos, bajo cualquier medición, son iguales. Pero, por razones de localización del trabajo, deciden mudarse a otra zona, rentándose mutuamente su hogar; al transparentarse el servicio de alquiler, automáticamente se registraría un aumento del ingreso y el gasto familiar de ambos y, por tanto, del país.

\section{Otros componentes}

El aspecto que más llama la atención del analista es el incremento del costo del transporte para el presupuesto de las familias. En 1976-77 un hogar típico destinaba el 6.1\% de su presupuesto al transporte y en 1984 el 6.3\%; esto es congruente con patrones internacionales, pero alcanzó un $16 \%$ en $1997-98$ y siguió subiendo hasta $17.3 \%$ en 2007 , aunque bajó ligeramente por debajo del 15\% en 2018.

Esto es una situación anómala y no parece humanamente razonable que una familia tenga que destinar una porción tan grande del fruto de su esfuerzo a cubrir los gastos de su movilización, más aún cuando los mismos 
datos indican una creciente parte usada en comer fuera de la casa que, en alguna medida, debería ayudar a reducir lo gastado en transporte; y todavía más, cuando la tecnología tiende a evitar los requerimientos de movilización, por las oportunidades de trabajo y recreación sin salir de casa.

Esto parece estar íntimamente vinculado con el patrón tecnológico que norma el sistema nacional de transporte de pasajeros, basado en el uso excesivo de medios individuales que resultan socialmente carísimos, como son el automóvil privado, el carro de concho (taxi colectivo con ruta predefinida) y el mototaxi. En particular, llama la atención pública sobre la enorme densidad de carros privados transitando por las calles, así como la de transporte público en automóvil, como efecto de la ausencia de un razonable sistema de transporte masivo.

Otros componentes que se convirtieron en importantes fueron el de comunicaciones que, de mantenerse oculto como parte de los servicios de la vivienda en las primeras encuestas, pasaron a convertirse en $4.5 \%$ del gasto familiar en 2018; los de educación y salud, que conjuntamente subieron de un $6.6 \%$ en la primera encuesta a $12.2 \%$ en la última; y diversos, que fue subiendo gradualmente, pero entre las últimas dos encuestas subió del $5 \%$ al $9.7 \%$. Dentro de este grupo destacan los bienes y servicios de cuidado personal.

\section{Probables cambios ocurridos en la estructura de consumo del país tras el COVID-19 o eventuales cambios futuros}

Las grandes conmociones socioeconómicas siempre han intensificado las tensiones sociales, acelerando cambios que de antemano se venían gestando o solían movilizar a la población. A nivel mundial, la pandemia generó una crisis económica de magnitudes desconocidas desde la Gran Depresión del decenio de 1930. La historia del siglo xx enseñó que todas las grandes crisis de la época conocida han aparejado grandes cambios:

- El fin de la Primera Guerra Mundial trajo el derrumbe de los imperios europeos.

- La Gran Depresión condujo a los esfuerzos de industrialización del Tercer Mundo, pero también contribuyó decisivamente al ascenso del Nazi-Fascismo y la Segunda Gran Guerra. 
- El fin de la Segunda Guerra Mundial generó el nuevo orden multilateral y una edad dorada del capitalismo.

- Hay una creciente coincidencia en que el capitalismo será diferente tras esta crisis, pero hay apreciaciones diferentes sobre cómo evolucionará la economía.

Antes de la pandemia el mundo se encontraba en plena efervescencia, como observa Massimo Morelli, profesor de Ciencias Políticas en la Universidad de Bocconi de Milán (García Vega, M. A., 2021).

En las cuatro décadas previas a la crisis del COVID-19, el capitalismo se hizo crecientemente injusto al interior de cada país, aunque paradójicamente más igualitario en términos geográficos, debido a que la economía creció y eso favoreció más a aquellas zonas del mundo con mayor concentración de pobreza -Asia y África- (Santana, I., 2019).

El problema de eso es que conduce a un sistema económico más injusto y que, a la larga, obligará al Estado a ocuparse de más cosas. Por ejemplo, los sistemas de seguridad social tendrán que ser adaptados para que los desocupados no se queden al margen del mercado. Esto indujo a gran parte del mundo académico a mirar al Estado a partir de otra dimensión. Es así como el discurso y la acción de los gobiernos, los organismos internacionales y la academia comenzaron a cambiar, y hasta intelectuales libres de toda sospecha de izquierdismo comienzan a plantearse la necesidad de una mayor presencia del Estado, la necesidad de una mayor cohesión social y cooperación entre las naciones.

De modo que ha surgido una creciente conciencia de que el enriquecimiento desmedido de las grandes fortunas vinculadas con los sectores ganadores, a costa del sufrimiento masivo del resto del mundo, demanda ideas novedosas para una intervención fiscal más progresista orientada a políticas redistributivas, a niveles nacionales y mundiales.

A este respecto se han pronunciado en el año 2021, tanto los presidentes de los Estados Unidos, de China y de la Unión Europea, organismos internacionales como el FMI, el Banco Mundial y comisiones de las Naciones Unidas y, finalmente, a eso apunta la propuesta de reforma fiscal internacional impulsada por la Organización para la Cooperación y el Desarrollo Económico (OECD), con el apoyo de 130 países para 
un impuesto mínimo de $15 \%$ a las ganancias de las multinacionales, así como la de un impuesto a las grandes fortunas en algunos países.

Sin embargo, al tiempo que segmentos mayores de las sociedades comenzaban a valorar más la presencia del Estado para afrontar problemas comunes, se hace evidente que tantas décadas de prédicas neoliberales fueron interiorizadas profundamente en los mundos académicos, políticos, empresariales y medios de comunicación. Por eso, no es raro ver todavía, en medio del drama, planteamientos pugnando por más neoliberalismo en materia económica, la convicción de que menos Estado y más bajos impuestos constituye la real solución; y que esto, en una especie de ejercicio de autoflagelación colectiva, genere amplias simpatías.

De todas formas, como la pandemia y la consecuente crisis va a dejar un mundo con gobiernos mucho más endeudados, una población empobrecida y programas públicos difíciles de desmontar por completo, alguien tendrá que cargar con los costos y es poco probable que el mundo vuelva a ser igual. Las condiciones económicas, sociales y políticas obligarán a repensar el mundo que nos hemos dado y a modificar grandes líneas de política.

A nivel de las personas y los hogares, es seguro que nada seguirá siendo exactamente igual: oportunidades perdidas, deseos aplazados, proyectos fracasados o postergados, seguirán afectando la memoria individual y colectiva, además de las pérdidas humanas. Esto estará pesando en los diversos países, incluyendo la República Dominicana.

Con gran probabilidad, algunos cambios que ocurrieron en el año 2020 serán de larga duración o permanentes. Muchas variaciones no han sido todavía debidamente medidas, sino que se irán percibiendo conforme se manifiestan en comportamientos diferentes de la población y de los sectores económicos.

Como se ha expuesto, los hábitos de consumo y la estructura de gastos de la población tiende a cambiar con el tiempo y las circunstancias, aun en tiempos normales. Pero la cosa es distinta cuando los tiempos dejan de ser "normales", como ha impuesto el COVID-19. Al principio de la pandemia, la idea que tenían los médicos, los organismos internacionales y los gobiernos era que se trataba de un fenómeno pasajero y que en un corto tiempo todo volvería a la normalidad anterior. 
Pero un confinamiento de largo plazo, con restricciones a las relaciones humanas, temor a la cercanía con el prójimo, alejamiento físico de la escuela y las universidades, trabajo a distancia y controles a los movimientos internacionales de las personas, tienden a provocar efectos mucho más marcados que lo habitual en los comportamientos humanos y en la estructura de gastos. Y con más razón cuando se producen cambios en muchos precios relativos o cuando la gente se acostumbra a ver la muerte relativamente de cerca.

La pandemia ha enseńado a infinidad de gente que puede vivir sin muchas cosas cuya posesión o consumo antes daba como algo natural. La canasta podría haber cambiado. Y si cambia abruptamente se produce un estremecimiento de la economía, pues la estructura productiva, el empleo, el comercio, la tributación, las importaciones, etc., se habían acomodado a esa canasta.

Negocios grandes, medianos, pequeños y micros nacieron y florecieron para dar respuesta a la canasta nacional y a las de otros países. Habrá que esperar por las mediciones, pues encuestas tan masivas y complejas como las ENGIH no suelen hacerse con una periodicidad reducida y es imposible ejecutarlas en momentos en que los jefes de hogar temen al acercamiento físico con encuestadores, pero los datos parciales provenientes de registros comenzarán a dar indicios.

Una aproximación podría irse viendo conforme crecen o decrecen los distintos sectores del PIB o los diversos renglones de importación. Pero los datos correspondientes al año 2020 e inicios del año 2021 son insuficientes, debido a que pueden estar reflejando, más que cambios estructurales de largo plazo, efectos de las mismas políticas aplicadas para contrarrestar la crisis sanitaria o atenuar la crisis económica; o bien al posterior rebote.

Algunos sectores del PIB crecieron en 2020 en medio de la crisis, a pesar de la pandemia o con la ayuda de las políticas públicas, como la agropecuaria, la salud, las comunicaciones y la intermediación financiera. En el año 2021 los sectores que más crecen, por efecto rebote, son justamente los que mayor deterioro sufrieron el año anterior y otros que han recibido un impulso por la propia pandemia. Tal es el caso de la construcción, las zonas francas, la industria manufacturera y el transporte (Banco Central de la República Dominicana, 2021). 
Crítica es la situación del sector turismo, que fue el que experimentó la caída más espectacular, debido principalmente al cierre. Este sector se redujo a su mínima expresión y, si bien comparado con el año 2020 los números son ahora positivos, las cifras indican que tomará algún tiempo la recuperación del nivel de actividad previo a la pandemia y mucho más que se vuelva a convertir en la mayor fuente de impulso del desarrollo económico dominicano.

Se anticipa la posibilidad de que el turismo como fenómeno de masas tenderá a agotarse y que habrá menos viajes de trabajo, privilegiando el trabajo a distancia. Que será más valorado un turismo de pequeños grupos, orientado a disfrutar la naturaleza y muy vinculado con la tecnología, por lo que será exigente en lo relativo a la disponibilidad de comunicaciones.

Observando el nivel y la estructura de las importaciones nacionales, en general todas bajaron en 2020, como bajó el comercio mundial, y la tendencia en el año 2021 es que nuevamente están subiendo (Dirección General de Aduanas). De su composición, de los datos publicados por la Dirección General de Aduanas lo más notorio que se deriva es un incremento en la importación de productos farmacéuticos en ambos años, lo cual no es para sorprender a nadie, y una fuerte reducción en las importaciones de combustibles en 2020 y posterior vuelta a aumentar en 2021, pero eso es básicamente explicado por los precios. Muchos otros productos experimentan altibajos, pero es demasiado temprano para avizorar tendencias a futuro.

Sin embargo, el nuevo modelo de vida impuesto por el COVID-19 tiene que haber comenzado a producir cambios drásticos, con mayor gasto en viviendas, comunicaciones, posiblemente muebles y artículos del hogar, pero pérdida relativa de los componentes prendas de vestir, hoteles, bares y restaurantes, transporte y otros como diversión y entretenimiento.

En el tiempo transcurrido hasta hoy, los datos internacionales muestran que los hogares se han volcado masivamente a la compra de bienes duraderos, incluyendo viviendas, vehículos, artículos del hogar, principalmente dispositivos electrónicos y de comunicaciones. Entre los servicios, los grandes ganadores son las comunicaciones, el sector financiero, el comercio por internet y de entrega a domicilio.

Como perdedores en la canasta de consumo se perciben las prendas y los accesorios de vestir, múltiples servicios como educación, viajes, espectáculos, restaurantes, entretenimiento, etc. Algo particular ocurre con los 
servicios de salud, siendo especialmente ganadores la industria farmacéutica, de equipos médicos y de diagnóstico, y la atención vinculada con enfermedades infectocontagiosas y la salud mental, pero en desmedro de la atención odontológica y la de otras especialidades médicas. Sin embargo, es previsible que en el mediano plazo el sector salud en su conjunto recobre una mayor importancia.

La pandemia ha obligado a las familias a permanecer en la vivienda mucho más tiempo del que era habitual. Esto ha venido provocando una creciente demanda de viviendas más espaciosas que permitan ampliar su rol tradicional, contar con espacios adecuados para reuniones y para socializar, incluso para recreación y para atender plataformas educativas y de salud mental, que serán importantes para ayudar en los procesos de adaptación con menores relaciones humanas presenciales. Obviamente, por razones de poder adquisitivo, esto no ocurrirá rápidamente, sino que será un proceso gradual que se irá adoptando primero en los estratos altos y se irá difundiendo gradualmente hacia abajo en la estratificación social.

Se anticipa también en la estructura de consumo una menor predilección por el lujo y más por el consumo sano y amigable al ambiente. También se prevé, y esto es importante para la canasta de consumo, la pérdida de importancia relativa de la vida en las grandes ciudades y la revitalización de las ciudades pequeñas y medianas.

\section{Referencias}

Banco Central de la República Dominicana. (1999). Encuesta Nacional de Gastos e Ingresos de los Hogares, Octubre 1997-Septiembre 1998. Tomo IV: Gastos e Ingresos de los Hogares, Santo Domingo, R. D.

Banco Central de la República Dominicana. (2018). Encuesta Nacional de Gastos e Ingresos de los Hogares (ENGIH) 2018. https://www. bancentral.gov.do/a/d/4796-engih-2018

Banco Central de la República Dominicana. (2021). Indicador mensual de actividad económica, 2021.

Del Rosario, G. (1984). Estructura del Consumo, Nutrición y Pobreza en la República Dominicana, 1984. Banco Central de la República Dominicana.

Del Rosario, P. J. (2021). El consumo de alimentos en la República Dominicana. IDIAF. 
Dirección General de Aduanas. https://www.aduanas.gob.do/estadisticas/ series-de-tiempo/

Engel, E. (1895), Die Lebenskosten Belguscher Arbeiter-Familien fruher und jetzt, Internacional Statistical. Institute Bulletin, 9, 1-74. Citado por Carugati, M. \& Berges, M., Ley de Engel y el Comportamiento de los Hogares en Argentina, Grupo de Economia Agraria. http://nulan. mdp.edu.ar/757/1/JDifInvEcon_2008_7_8-13.pdf

FMI. (2021). Actualización de las perspectivas de la Economía Mundial.

García Vega, M. A. (11 de septiembre de 2021). La ira amenaza la recuperación económica. El país. https://elpais.com/economia/2021-09-12/ la-ira-amenaza-la-recuperacion-economica.html

Santana, I. (2019). La perspectiva de la República Dominicana en el marco de las cambiantes tendencias mundiales. Ministerio de Economía, Planificación y Desarrollo, Santo Domingo. 
